Received: 11 May 2018

Accepted: 8 March 2019

Published online: 25 March 2019
SCIENTIFIC REP
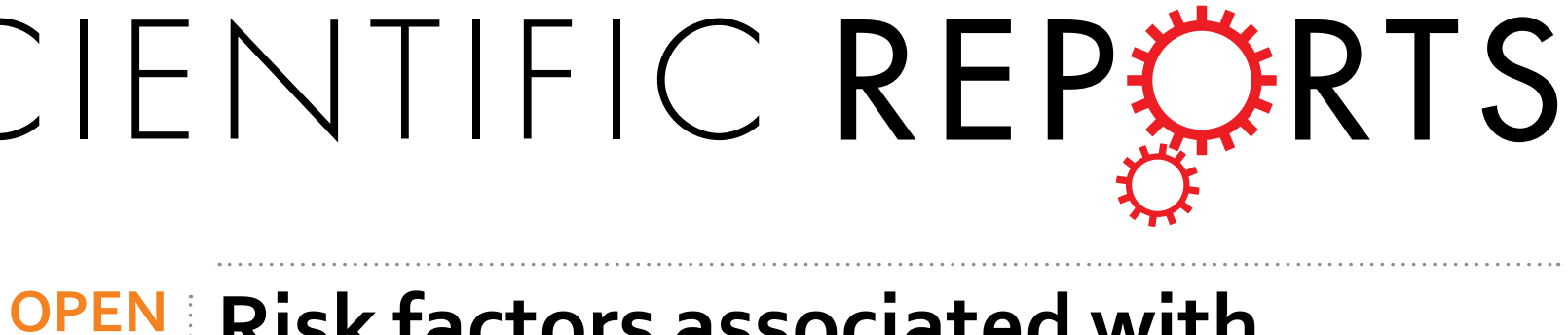

\section{Risk factors associated with} locoregional failure and estimation of survival after curative resection for patients with distal bile duct

\section{cancer}

\author{
Jung Ho $\mathrm{Im}^{1}$, Joon Seong Park², Dong Sup Yoon², Dong Ki Lee ${ }^{3}$, Jun Won Kim4 \& Ik Jae Lee $\mathbb{C}^{4}$
}

Our aim was to identify the risk factors associated with locoregional recurrence in resected distal bile duct cancer ( $D B D C$ ), and to determine the subgroup that may benefit from adjuvant radiotherapy. Between 2001 and 2013, we retrospectively analyzed 93 patients with DBDC who had undergone curative resection. Patients who received adjuvant radiotherapy were excluded. The 3-year locoregional failure-free survival (LRFFS) and overall survival (OS) rates for all patients were $50.7 \%$, and $53.2 \%$, respectively. On multivariate analysis, the preoperative carcinoembryonic antigen (CEA) level, resection margin, histologic grade, $\mathrm{T}$ stage, and $\mathrm{N}$ stage were significant prognostic factors for LRFFS. Locoregional recurrence was observed in more than $78 \%$ of the patients who underwent R1 resection and were node-positive, and the 3-year LRFFS rate was $19.3 \%$. The 3-year LRFFS rate was $46.9 \%$ in the patients who underwent $R 0$ resection and were node-negative with more than 2 risk factors (preoperative CEA level $\geq 5 \mathrm{ng} / \mathrm{mL}$, poorly differentiated histologic grade, and T3 stage). On multivariate analysis for OS, patients with more than 2 risk factors showed a 7-fold higher risk of death, compared with patients with 1 or no risk factor. The important risk factors of locoregional failure in patients with DBDC who underwent resection were R1 resection and positive lymph nodes. Adjuvant radiotherapy should be considered for these patients to improve the oncologic outcomes. Patients undergoing selective $\mathrm{R} O$ resection and those with node-negative status and multiple locoregional failure risk factors may benefit from adjuvant radiotherapy.

Curative resection is the treatment of choice for distal bile duct cancer (DBDC). However, the prognosis of DBDC remains unsatisfactory even after curative resection, with the 5-year overall survival (OS) rate at only approximately $25-48 \%{ }^{1-7}$. One study showed that $52 \%$ of patients who underwent resection without adjuvant treatment had disease recurrence ${ }^{6}$. The prognosis after recurrence is dismal, with a median OS of only 6-9 months ${ }^{1-3}$.

Although the efficacy of adjuvant treatment after curative resection remains controversial, adjuvant treatment is commonly used to improve the poor prognosis of DBDC. Adjuvant radiotherapy has been recommended to improve locoregional control and has increased survival ${ }^{4-6,8-10}$.

A better understanding of locoregional recurrence after resection for DBDC is essential in implementing radiotherapy and improving surgical outcomes. However, because of the rarity of DBDC ${ }^{11,12}$, the risk factors for locoregional recurrence have not been well established and research on prognostic factors influencing locoregional failure-free survival (LRFFS) is limited. Therefore, the aim of this study was to focus on locoregional recurrence after curative resection of DBDC. We analyzed the risk factors associated with locoregional recurrence in

${ }^{1}$ Department of Radiation Oncology, CHA Bundang Medical Center, CHA University School of Medicine, Seongnam, Korea. ${ }^{2}$ Pancreatobiliary Cancer Clinic, Department of Surgery, Gangnam Severance Hospital, Yonsei University College of Medicine, Seoul, Korea. ${ }^{3}$ Department of Internal Medicine, Gangnam Severance Hospital, Yonsei University College of Medicine, Seoul, Korea. ${ }^{4}$ Departments of Radiation Oncology, Gangnam Severance Hospital, Yonsei University College of Medicine, Seoul, Korea. Correspondence and requests for materials should be addressed to J.S.P. (email: jspark330@yuhs.ac) or I.J.L. (email: ikjae412@yuhs.ac) 
patients undergoing surgical resection for DBDC and investigated the subgroup of patients who may benefit the most from adjuvant radiotherapy.

\section{Results}

Patient characteristics. The characteristics of the 93 included patients are listed in Table 1 . The median age was 67 years (range, $34-81$ years). The median preoperative and postoperative carbohydrate antigen (CA) 19-9 levels were $96.6 \mathrm{U} / \mathrm{mL}$ and 14.8 (range, $1.8-4529$ ) $\mathrm{U} / \mathrm{mL}$, respectively and the median preoperative carcinoembryonic antigen (CEA) level was 2.5 (range, $0.3-32.1$ ) ng/mL. The preoperative CA 19-9 level of 1 patient was higher than $20040 \mathrm{U} / \mathrm{mL}$. Nineteen patients underwent segmental resection of bile duct; 74 underwent pylorus-preserving pancreatoduodenectomy. After surgical resection, R0 resection was achieved in 79 patients (84.9\%), and R1 resection in 14 patients (15.1\%). Metastatic lymph nodes (LNs) were found in $39.8 \%$ of patients.

Survival analysis and patterns of failure. The median follow-up was 46 months (range, $8-155$ months) for the surviving patients. Of the surviving patients, $13(13 / 45,26.7 \%)$ were followed up for 3 years or less. The median OS was 47 months. The 3 -year OS and LRFFS rates were $53.2 \%$ and $50.7 \%$, respectively.

During follow-up, 56 patients $(60.2 \%)$ were diagnosed with recurrence, and locoregional recurrence was the dominant type of failure. Locoregional recurrences were the first event in 50 patients $(53.8 \%)$, and distant relapse occurred in 40 patients (43.0\%). Thirty-four patients (36.6\%) had both locoregional relapse and distant metastasis. The liver was the most common site for primary metastatic recurrence.

Risk factors related to locoregional failure-free survival and overall survival. Initial locoregional recurrence was more frequent in patients with preoperative CA $19-9$ level $>37 \mathrm{U} / \mathrm{mL}$, positive resection margin (RM), T3 stage, and positive LNs (Table 1). Initial locoregional recurrence occurred in $85.7 \%$ and $78.4 \%$ of the patients with positive RM and positive LNs, respectively. Initial locoregional recurrence occurred in 23 (79.3\%) of 29 patients who had positive LNs and negative RM. The preoperative and postoperative CA 19-9 level, RM, lymphovascular invasion, T stage, and N stage were significant prognostic factors for LRFFS $(\mathrm{p}<0.05)($ Table 2). Multivariate analysis showed that preoperative CEA level, RM, histologic grade, $\mathrm{T}$ stage, and $\mathrm{N}$ stage were independent prognostic factors for LRFFS ( $p<0.05$ ) (Table 3 ).

All patients were divided into 3 locoregional recurrence risk groups according to risk factors (Table 4). As locoregional recurrence occurred in more than $78 \%$ of the patients with positive RM and LNs, these patients were defined as the high-risk group for locoregional recurrence (group $1, n=42$ ). The patients, with negative $\mathrm{RM}$ and LNs, were divided into 2 groups according to the preoperative CEA level, histologic grade, and T stage considering the 5 independent prognostic factors for LRFFS on multivariate analysis. The patients, who had more than 2 risk factors considering preoperative CEA level $\geq 5 \mathrm{ng} / \mathrm{mL}$, poorly differentiated histologic grade, and T3 stage, were defined as group $2(n=12)$, and those who had less than 1 risk factor were defined as group $3(n=39)$. The locoregional recurrence rates of groups 1,2 , and 3 were $81.0 \%, 50.0 \%$, and $25.6 \%$, respectively (Table 1 ). The 3 -year LRFFS for groups 1, 2, and 3 were 19.3\%, 46.9\%, and 84.2\%, respectively (Fig. 1). LRFFS was significantly different among the three groups $(\mathrm{p}<0.05)$ (Table 2). Moreover, there were significant differences in LRFFS between groups 1 and 2 , groups 2 and 3 , and groups 1 and $3(\mathrm{p}<0.05)$.

The results of univariate analysis of OS are summarized in Table 2. Univariate analysis showed that preoperative and postoperative CA 19-9 levels, RM, lymphovascular invasion, T stage, $\mathrm{N}$ stage, and locoregional recurrence risk group were significantly associated with OS $(\mathrm{p}<0.05)$. For multivariate analysis, the analysis was conducted by substituting locoregional recurrence risk group. On multivariate analysis, locoregional recurrence risk group, postoperative CA19-9 level, and lymphovascular invasion were significantly associated with OS $(\mathrm{P}<0.05)$ (Table 3 ). The patients in groups 1 and 2 showed approximately 8 -fold and 7 -fold increased risks of death, respectively, as compared with group $3(\mathrm{p}<0.05)$.

\section{Discussion}

The role of adjuvant radiotherapy has not been well established. The literature regarding the benefit of adjuvant radiotherapy for DBDC is inconsistent ${ }^{4-6,8,9,13}$. For the appropriate selection of radiotherapy, risk factors associated with locoregional failure are thought to be an integral part of DBDC management. Several studies reported that $47-66 \%$ of patients with DBDC still experienced disease recurrence after undergoing surgical resection, and the initial locoregional recurrence rate was $26-65 \%^{1-3,6,7,14,15}$. This study was conducted in patients with DBDC who underwent resection but did not receive adjuvant radiotherapy, and the initial locoregional recurrence rate was 53.8\%. Accordingly, adjuvant radiotherapy should be administered for selected patients with DBDC who undergo resection and are at a higher risk of locoregional failure regardless of whether the dominant type of failure is a locoregional recurrence or distant metastasis.

In this study, RM and LNs were found to be high risk factors of locoregional recurrence. However, Kim et al. found that $\mathrm{N}$ stage and perineural invasion were significantly associated with locoregional control in patients with resected extrahepatic biliary tract cancer $^{16}$, while Choi et al. reported that the LN status and RM status were not significant factors for locoregional recurrence, and that poorly differentiated histologic grade was an independent prognostic factor for locoregional recurrence ${ }^{1}$. The discrepancy in the risk factors among the studies can be partly explained by the fact that radiotherapy is usually performed in patients with positive RM. In other words, as adjuvant radiotherapy for patients with positive RM might be effective in controlling microscopic residual tumors, the RM may not be a risk factor of locoregional recurrence. A recent meta-analysis demonstrated that adjuvant chemotherapy or chemoradiotherapy significantly improved the survival of patients with biliary tract cancer with positive LNs or positive $\mathrm{RM}^{9}$. Our previous study showed that adjuvant chemoradiotherapy improved the clinical outcomes of patients with resected extrahepatic bile duct cancer, particularly patients who underwent R1 resection ${ }^{8}$. Kim et al. reported that adjuvant chemotherapy or chemoradiotherapy was associated with a significant OS 


\begin{tabular}{|c|c|c|c|c|}
\hline \multirow[b]{3}{*}{ Variable } & \multicolumn{4}{|c|}{ Number of patients (\%) } \\
\hline & & Loco-regi & ecurrence & \multirow[b]{2}{*}{ p-value } \\
\hline & & Yes & No & \\
\hline Age (years) & & & & 0.277 \\
\hline$\leq 65$ & $35(40.9)$ & $23(60.5)$ & $15(39.5)$ & \\
\hline$>65$ & $55(59.1)$ & $27(49.1)$ & $28(50.9)$ & \\
\hline Sex & & & & 0.108 \\
\hline Male & $59(63.4)$ & $28(47.5)$ & $31(52.5)$ & \\
\hline Female & $34(36.6)$ & $22(64.7)$ & $12(35.3)$ & \\
\hline Preoperative CA 19-9(U/mL) & & & & 0.011 \\
\hline$<37$ & $25(26.9)$ & $8(32.0)$ & $17(68.0)$ & \\
\hline$\geq 37$ & $68(73.1)$ & $42(61.8)$ & $26(38.2)$ & \\
\hline Postoperative CA 19-9 (U/mL) & & & & 0.337 \\
\hline$<37$ & $81(87.1)$ & $42(51.9)$ & $39(48.1)$ & \\
\hline$\geq 37$ & $12(12.9)$ & $8(66.7)$ & $4(33.3)$ & \\
\hline Preoperative CEA $(\mathrm{ng} / \mathrm{mL})$ & & & & 0.255 \\
\hline$<5$ & $73(78.5)$ & $37(50.7)$ & $36(49.3)$ & \\
\hline$\geq 5$ & $20(21.5)$ & $13(65.0)$ & $7(35.0)$ & \\
\hline Surgical procedure & & & & 0.912 \\
\hline PPPD & $74(79.6)$ & $40(54.1)$ & $34(45.9)$ & \\
\hline Segmental resection of bile duct & $19(20.4)$ & $10(52.6)$ & $9(47.4)$ & \\
\hline Resection margin & & & & 0.009 \\
\hline R0 & $79(84.9)$ & $38(48.1)$ & $41(51.9)$ & \\
\hline R1 & $14(15.1)$ & $12(85.7)$ & $2(14.3)$ & \\
\hline Histologic grade & & & & 0.255 \\
\hline $\mathrm{WD} / \mathrm{MD}$ & $73(78.5)$ & $37(50.7)$ & $36(49.3)$ & \\
\hline PD & $20(21.5)$ & $13(65.0)$ & $7(35.0)$ & \\
\hline Lymphovascular invasion & & & & 0.161 \\
\hline No & $67(72.0)$ & $33(49.3)$ & $34(50.7)$ & \\
\hline Yes & $26(28.0)$ & $17(65.4)$ & $9(34.6)$ & \\
\hline Perineural invasion & & & & 0.981 \\
\hline No & $28(30.1)$ & $15(53.6)$ & $13(46.4)$ & \\
\hline Yes & $65(69.9)$ & $35(53.8)$ & $30(46.2)$ & \\
\hline T stage (AJCC 7th edition) & & & & 0.011 \\
\hline $\mathrm{T} 1-2$ & $41(44.1)$ & $16(39.0)$ & $25(61.0)$ & \\
\hline T3 & $52(55.9)$ & $34(65.4)$ & $18(34.6)$ & \\
\hline $\mathrm{N}$ stage (AJCC 7th edition) & & & & $<0.001$ \\
\hline N0 & $56(60.2)$ & $21(37.5)$ & $35(62.5)$ & \\
\hline N1 & $37(39.8)$ & $29(78.4)$ & $8(21.6)$ & \\
\hline $\mathrm{N}$ stage (AJCC 8th edition) & & & & 0.001 \\
\hline No & $56(60.2)$ & $21(37.5)$ & $35(62.5)$ & \\
\hline $\mathrm{N} 1$ & $29(31.2)$ & $23(79.3)$ & $6(20.7)$ & \\
\hline $\mathrm{N} 2$ & $8(8.6)$ & $6(75.0)$ & $2(25.0)$ & \\
\hline Adjuvant chemotherapy & & & & 0.619 \\
\hline No & $45(48.4)$ & $23(51.1)$ & $22(48.9)$ & \\
\hline Yes & $48(51.6)$ & $27(56.3)$ & $21(43.8)$ & \\
\hline Locoregional recurrence risk group & & & & $<0.001$ \\
\hline Group 1 & $42(45.2)$ & $34(81.0)$ & $8(19.0)$ & \\
\hline Group 2 & $12(12.9)$ & $6(50.0)$ & $6(50.0)$ & \\
\hline Group 3 & $42(45.2)$ & $10(25.6)$ & $29(74.4)$ & \\
\hline
\end{tabular}

Table 1. Patient characteristics of all patients and relationship between patient characteristics and locoregional recurrence. CA, carbohydrate antigen; CEA, carcinoembryonic antigen; PPPD, pylorus-preserving pancreaticoduodenectomy; WD, well differentiated; $\mathrm{MD}$, moderately differentiated; $\mathrm{PD}$, poorly differentiated; AJCC, American Joint Committee on Cancer Group 1 was defined as patients with positive resection margin or positive lymph nodes. Group 2 was defined as patients with negative resection margin and negative lymph nodes who possessed 2 or more risk factors (preoperative CEA level $\geq 5 \mathrm{ng} / \mathrm{mL}$, poorly differentiated histologic grade, and T3 stage). Group 3 was defined as patients with negative resection margin and negative lymph nodes with 1 or no risk factor (preoperative CEA level $\geq 5 \mathrm{ng} / \mathrm{mL}$, poorly differentiated histologic grade, and T3 stage). 


\begin{tabular}{|c|c|c|c|c|}
\hline \multirow[b]{2}{*}{ Prognostic factor } & \multicolumn{4}{|c|}{ 3-y Survival rate (\%) } \\
\hline & LRFFS & p-value & OS & p-value \\
\hline Age (years) & & 0.276 & & 0.493 \\
\hline$\leq 65$ & 43.3 & & 48.5 & \\
\hline$>65$ & 55.9 & & 56.5 & \\
\hline Sex & & 0.167 & & 0.636 \\
\hline Male & 56.1 & & 60.5 & \\
\hline Female & 41.5 & & 39.2 & \\
\hline Preoperative CA 19-9 (U/mL) & & 0.019 & & 0.014 \\
\hline$<37$ & 70.2 & & 78.6 & \\
\hline$\geq 37$ & 43.6 & & 44.6 & \\
\hline Postoperative CA 19-9 (U/mL) & & 0.001 & & $<0.001$ \\
\hline$<37$ & 54.4 & & 58.6 & \\
\hline$\geq 37$ & 27.8 & & 16.7 & \\
\hline Preoperative CEA (ng/mL) & & 0.087 & & 0.056 \\
\hline$<5$ & 54.2 & & 57.2 & \\
\hline$\geq 5$ & 37.9 & & 39.4 & \\
\hline Surgical procedure & & 0.853 & & 0.811 \\
\hline PPPD & 50.5 & & 53.5 & \\
\hline Segmental resection of bile duct & 51.7 & & 52.1 & \\
\hline Resection margin & & $<0.001$ & & $<0.001$ \\
\hline R0 & 56.5 & & 60.2 & \\
\hline $\mathrm{R} 1$ & 15.9 & & 14.3 & \\
\hline Histologic grade & & 0.104 & & 0.268 \\
\hline $\mathrm{WD} / \mathrm{MD}$ & 54.1 & & 55.8 & \\
\hline PD & 38.2 & & 44.4 & \\
\hline Lymphovascular invasion & & 0.008 & & $<0.001$ \\
\hline No & 57.2 & & 64.1 & \\
\hline Yes & 33.3 & & 25.2 & \\
\hline Perineural invasion & & 0.954 & & 0.808 \\
\hline No & 47.2 & & 50.8 & \\
\hline Yes & 52.2 & & 54.2 & \\
\hline T stage (AJCC 7th edition) & & 0.003 & & 0.002 \\
\hline $\mathrm{T} 1-2$ & 69.6 & & 69.7 & \\
\hline T3 & 35.1 & & 40.0 & \\
\hline $\mathrm{N}$ stage (AJCC 7th edition) & & $<0.001$ & & $<0.001$ \\
\hline No & 70.4 & & 69.8 & \\
\hline $\mathrm{N} 1$ & 19.3 & & 29.0 & \\
\hline N stage (AJCC 8th edition) & & $<0.001$ & & $<0.001$ \\
\hline N0 & 70.4 & & 69.8 & \\
\hline $\mathrm{N} 1$ & 21.6 & & 34.1 & \\
\hline $\mathrm{N} 2$ & 25.0 & & 12.5 & \\
\hline Adjuvant chemotherapy & & 0.404 & & 0.164 \\
\hline No & 58.6 & & 63.0 & \\
\hline Yes & 43.1 & & 44.4 & \\
\hline Locoregional recurrence risk group & & $<0.001$ & & $<0.001$ \\
\hline Group 1 & 19.3 & & 28.0 & \\
\hline Group 2 & 46.9 & & 45.7 & \\
\hline Group 3 & 84.2 & & 83.5 & \\
\hline
\end{tabular}

Table 2. Univariate analysis of prognostic factors for locoregional failure-free survival and overall survival. CA, carbohydrate antigen; CEA, carcinoembryonic antigen; PPPD, pylorus-preserving pancreaticoduodenectomy; WD, well differentiated; MD, moderately differentiated; PD, poorly differentiated; AJCC, American Joint Committee on Cancer; LRFFS, locoregional failure-free survival; OS, overall survival. Group 1 was defined as patients with positive resection margin or positive lymph nodes. Group 2 was defined as patients with negative resection margin and negative lymph nodes who possessed 2 or more risk factors (preoperative CEA level $\geq 5 \mathrm{ng} / \mathrm{mL}$, poorly differentiated histologic grade, and T3 stage). Group 3 was defined as patients with negative resection margin and negative lymph nodes with 1 or no risk factor (preoperative CEA level $\geq 5 \mathrm{ng} / \mathrm{mL}$, poorly differentiated histologic grade, and T3 stage). 


\begin{tabular}{|l|l|l|l|l|}
\hline \multirow{2}{*}{ Variable } & \multicolumn{2}{l}{ LRFFS } & OS \\
\cline { 2 - 5 } & HR $(95 \%$ CI $)$ & p-value & HR (95\% CI) & p-value \\
\hline Preoperative CEA $(\geq 5 \mathrm{ng} / \mathrm{mL})$ & $2.169(1.111-4.237)$ & 0.023 & - & - \\
\hline Resection margin (R1) & $3.912(1.835-8.343)$ & $<0.001$ & - & - \\
\hline Histologic grade (PD) & $2.208(1.125-4.337)$ & 0.021 & - & - \\
\hline T stage (T3, AJCC 7th edition) & $3.473(1.788-6.747)$ & $<0.001$ & - & - \\
\hline N stage (N1, AJCC 7th edition) & $4.386(2.278-8.444)$ & $<0.001$ & - & - \\
\hline Locoregional recurrence risk group 3 & - & - & $6.927(2.120-22.632)$ & 0.001 \\
\hline vs Group 2 & - & - & $8.258(3.345-20.388)$ & $<0.001$ \\
\hline vs Group 1 & - & - & $2.183(0.926-5.144)$ & 0.074 \\
\hline Preoperative CA 19-9 $(\geq 37 \mathrm{U} / \mathrm{mL})$ & - & - & $2.489(1.199-5.163)$ & 0.014 \\
\hline Postoperative CA 19-9 $(\geq 37 \mathrm{U} / \mathrm{mL})$ & - & - & $2.339(1.247-4.387)$ & 0.008 \\
\hline Lymphovascular invasion $($ Positive) & - & & & \\
\hline
\end{tabular}

Table 3. Multivariate analysis of prognostic factors for locoregional failure-free survival and overall survival. CEA, carcinoembryonic antigen; PD, poorly differentiated; AJCC, American Joint Committee on Cancer; CA, carbohydrate antigen; LRFFS, locoregional failure-free survival; OS, overall survival; HR, hazard ratio; CI, confidence interval. Group 1 was defined as patients with positive resection margin or positive lymph nodes. Group 2 was defined as patients with negative resection margin and negative lymph nodes who possessed 2 or more risk factors (preoperative CEA level $\geq 5 \mathrm{ng} / \mathrm{mL}$, poorly differentiated histologic grade, and T3 stage). Group 3 was defined as patients with negative resection margin and negative lymph nodes with 1 or no risk factor (preoperative CEA level $\geq 5 \mathrm{ng} / \mathrm{mL}$, poorly differentiated histologic grade, and T3 stage).

\begin{tabular}{|l|l|l|}
\hline Group & Definition & $\begin{array}{l}\text { Number of } \\
\text { Patients }\end{array}$ \\
\hline Group1 & Patients with R1 resection or positive lymph node & 42 \\
\hline Group2 & $\begin{array}{l}\text { R0 resection and N0 patients who possessed two to three risk factors (preoperative CEA level } \geq 5[\mathrm{ng} / \mathrm{mL}], \\
\text { poorly differentiated histologic grade, and T3 stage) }\end{array}$ & 12 \\
\hline Group3 & $\begin{array}{l}\text { R0 resection and N0 patients who did not exhibit any risk factors or had one risk factor (preoperative CEA } \\
\text { level } \geq 5[\mathrm{ng} / \mathrm{mL}], \text { poorly differentiated histologic grade, and T3 stage) }\end{array}$ & 39 \\
\hline
\end{tabular}

Table 4. Locoregional recurrence risk groups according to locoregional recurrence risk factors. CEA, carcinoembryonic a ntigen.

advantage in patients with LN-positive R0-resected DBDC ${ }^{6}$. Therefore, adjuvant radiotherapy should be considered for locoregional control of RM-positive or LN-positive DBDC.

The benefit of adjuvant radiotherapy remains unclear for patients with DBDC and negative LNs treated with $\mathrm{R} 0$ resection. In this study, the locoregional recurrences rate was $25.6 \%$ in patients with 1 or no risk factor among preoperative CEA level $\geq 5 \mathrm{ng} / \mathrm{mL}$, poorly differentiated histologic grade, and T3 stage. Therefore, we believe that adjuvant radiotherapy may not improve the clinical outcomes of these patients. However, locoregional recurrence occurred in $50.0 \%$ of the patients who underwent R0 resection and had negative LNs with more than 2 risk factors, and the 5-year LRFFS was $46.9 \%$; thus, patients with more than 2 risk factors showed a 7 -fold higher risk for death compared with those with 1 or no risk factors on multivariate analysis for OS. Adjuvant radiotherapy may be helpful to increase survival outcome for some of these patients. Nevertheless, further studies should be performed to determine whether adjuvant radiotherapy can actually improve clinical outcomes of these patients.

There are several limitations of this study. First, this was a nonrandomized and retrospective study, and unrecognized bias could be present. As this study was conducted at a single center, and the patients treated with adjuvant radiotherapy were excluded, selection bias may have affected the results. Further, 13 surviving patients were followed up for 3 years or less which may also have generated selection bias. Additionally, because the present study involved a limited number of patients, the results of this study must be confirmed in larger studies in the future. Finally, CA 19-9 and CEA levels could show a false positive or negative result. Therefore, caution is needed when evaluating the recurrence or prognosis of the patients ${ }^{17}$.

In conclusion, R1 resection and positive LNs were important prognostic factors for locoregional recurrence in patients with DBDC. Therefore, we recommend adjuvant radiotherapy with curative resection for patients with DBDC treated with R1 resection or those who have positive LNs. Adjuvant radiotherapy for patients treated with R0 resection and those with negative LNs who exhibit multiple locoregional risk factors may reduce locoregional failure, which translates into OS benefits. Future well-designed, randomized, controlled studies are necessary to clarify the role of adjuvant radiotherapy.

\section{Methods}

The study protocol conformed to the ethical guidelines of the 1975 Declaration of Helsinki, as revised in 1983, and was approved by Institutional Review Board of Severance Hospital. The patient records/information was anonymized and de-identified prior to analysis, and informed consent was not obtained from each participant. After Institutional Review Board approval, we retrospectively reviewed the records of all patients with DBDC 


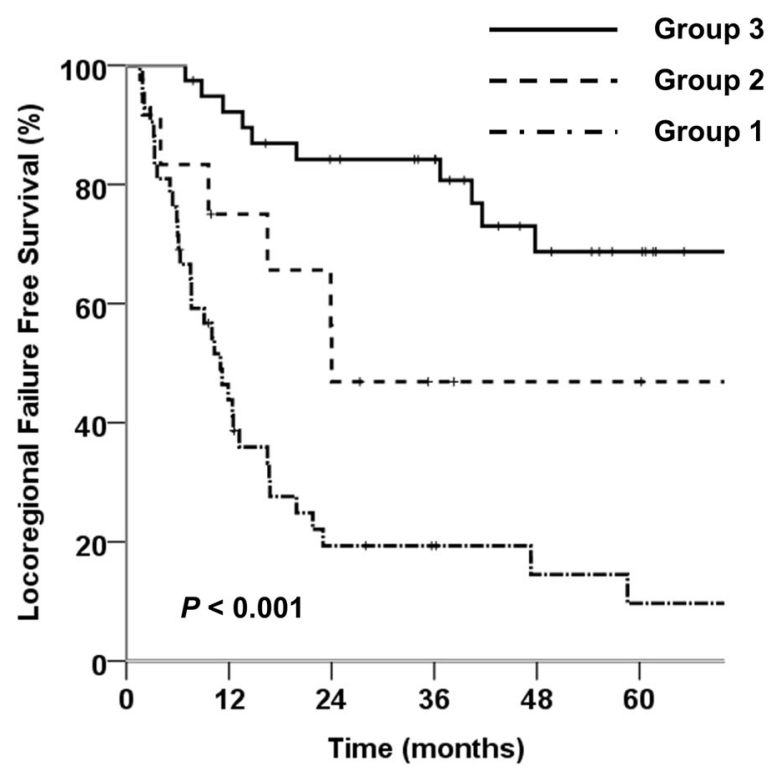

Figure 1. Comparison of locoregional failure-free survival curves according to locoregional recurrence risk groups. Group 1 was defined as patients with positive resection margin or positive lymph nodes. Group 2 was defined as patients with negative resection margin and negative lymph nodes who possessed 2 or more risk factors (preoperative CEA level $\geq 5 \mathrm{ng} / \mathrm{mL}$, poorly differentiated histologic grade, and T3 stage). Group 3 was defined as patients with negative resection margin and negative lymph nodes with 1 or no risk factor (preoperative CEA level $\geq 5 \mathrm{ng} / \mathrm{mL}$, poorly differentiated histologic grade, and T3 stage).

adenocarcinoma who underwent curative surgical resection between January 2001 and December 2013 . The inclusion criteria were no distant metastasis and an Eastern Cooperative Oncology Group performance status of $\leq 2$. Patients were excluded if they received neoadjuvant therapy or adjuvant radiotherapy; patients with ampullary and duodenal carcinomas were also excluded from this study. Finally, 93 patients with DBDC, confirmed by pathological diagnosis after surgical resection, were included in this study. The primary outcome was the 3-year LRFFS in patients undergoing surgical resection for distal bile duct cancer; the secondary outcomes were OS and patterns of failure.

The type of surgery selected was determined according to the location of the tumor: if the tumor was located in the distal bile duct, pancreaticoduodenectomy was performed; if the tumor was located in the mid portion of the bile duct, segmental resection and hepaticojejunostomy were performed. All patients underwent dissection of the LNs in the hepatoduodenal ligament, as well as common hepatic artery and celiac axis LNs. Patients with histologically positive LNs or poorly differentiated DBDC considering the histologic grade were usually treated with adjuvant chemotherapy. Adjuvant 5-fluorouracil-based or gemcitabine-based chemotherapy was administered at the physician's discretion.

The disease stage for all patients was determined according to the 7th edition of the American Joint Committee on Cancer System. Positive margins were defined as the presence of at least 1 cancer cell at the RM on microscopic examination. CA 19-9 level of $37 \mathrm{U} / \mathrm{mL}$ and CEA level of $5 \mathrm{ng} / \mathrm{mL}$ was defined as a cutoff value for normal level 2,6,8,15-17.

Patients were followed-up 1 month after the operation and every 3 months during the first 12 months, and then every 6 months beyond the first year. Patients underwent measurements of CA 19-9 and CEA, and underwent computed tomography. The postoperative CA 19-9 level was measured between 1 week and 1 month after surgical resection. Tumor recurrences were confirmed using radiological imaging techniques and pathological studies of histological specimens, with the majority being made by radiological evaluation, including computed tomography and positron emission tomography. Recurrence was also confirmed pathologically by biopsy or via radiological findings. In this study, only the initial recurrence was evaluated. Locoregional recurrence was defined as recurrence in the primary tumor bed and regional lymphatic areas. All other recurrences were considered distant metastasis.

Survival was calculated from the date of surgical resection. All events were measured from the date of surgery to the date of recurrence. A chi-square test or Fisher exact test was used for comparison of categorical variables between groups. Survival was estimated by using the Kaplan-Meier method and compared with the log-rank test. Multivariate analysis was performed using a Cox proportional hazards model, and the hazard ratio with a $95 \%$ confidence interval was determined. We performed a multivariate analysis using backwards elimination to stay in the model. A p-value of $<0.05$ indicated statistical significance.

\section{References}

1. Choi, S. B. et al. Disease recurrence patterns and analysis of clinicopathological prognostic factors for recurrence after resection for distal bile duct cancer. Am Surg 81, 289-296 (2015).

2. Choi, S. B., Park, S. W., Kim, K. S., Choi, J. S. \& Lee, W. J. The survival outcome and prognostic factors for middle and distal bile duct cancer following surgical resection. J Surg Oncol 99, 335-342, https://doi.org/10.1002/jso.21238 (2009). 
3. Komaya, K. et al. Recurrence after resection with curative intent for distal cholangiocarcinoma. Br J Surg 104, 426-433, https://doi. org/10.1002/bjs.10452 (2017).

4. Todoroki, T. et al. Treatment strategy for patients with middle and lower third bile duct cancer. Br J Surg 88, 364-370, https://doi. org/10.1046/j.1365-2168.2001.01685.x (2001).

5. Hughes, M. A. et al. Adjuvant concurrent chemoradiation for adenocarcinoma of the distal common bile duct. Int J Radiat Oncol Biol Phys 68, 178-182, https://doi.org/10.1016/j.ijrobp.2006.11.048 (2007).

6. Kim, Y. S. et al. Role of adjuvant therapy after R0 resection for patients with distal cholangiocarcinoma. Cancer Chemother Pharmacol 77, 979-985, https://doi.org/10.1007/s00280-016-3014-x (2016).

7. Chung, Y. J., Choi, D. W., Choi, S. H., Heo, J. S. \& Kim, D. H. Prognostic factors following surgical resection of distal bile duct cancer. J Korean Surg Soc 85, 212-218, https://doi.org/10.4174/jkss.2013.85.5.212 (2013).

8. Im, J. H. et al. Surgery Alone Versus Surgery Followed by Chemotherapy and Radiotherapy in Resected Extrahepatic Bile Duct Cancer: Treatment Outcome Analysis of 336 Patients. Cancer Res Treat 48, 583-595, https://doi.org/10.4143/crt.2015.091 (2016).

9. Horgan, A. M., Amir, E., Walter, T. \& Knox, J. J. Adjuvant therapy in the treatment of biliary tract cancer: a systematic review and meta-analysis. J Clin Oncol 30, 1934-1940, https://doi.org/10.1200/jco.2011.40.5381 (2012).

10. Kim, M. Y., Kim, J. H., Kim, Y. \& Byun, S. J. Postoperative radiotherapy appeared to improve the disease free survival rate of patients with extrahepatic bile duct cancer at high risk of loco-regional recurrence. Radiat Oncol J 34, 297-304, https://doi.org/10.3857/ roj.2016.01879 (2016).

11. Cardinale, V. et al. Intra-hepatic and extra-hepatic cholangiocarcinoma: New insight into epidemiology and risk factors. World J Gastrointest Oncol 2, 407-416, https://doi.org/10.4251/wjgo.v2.i11.407 (2010).

12. Jung, K. W. et al. Cancer Statistics in Korea: Incidence, Mortality, Survival, and Prevalence in 2014. Cancer Res Treat 49, 292-305, https://doi.org/10.4143/crt.2017.118 (2017).

13. Vern-Gross, T. Z. et al. Survival outcomes in resected extrahepatic cholangiocarcinoma: effect of adjuvant radiotherapy in a surveillance, epidemiology, and end results analysis. Int J Radiat Oncol Biol Phys 81, 189-198, https://doi.org/10.1016/j. ijrobp.2010.05.001 (2011).

14. Kwon, H. J., Kim, S. G., Chun, J. M., Lee, W. K. \& Hwang, Y. J. Prognostic factors in patients with middle and distal bile duct cancers. World J Gastroenterol 20, 6658-6665, https://doi.org/10.3748/wjg.v20.i21.6658 (2014).

15. Koo, T. R. et al. Patterns of failure and prognostic factors in resected extrahepatic bile duct cancer: implication for adjuvant radiotherapy. Radiat Oncol J 32, 63-69, https://doi.org/10.3857/roj.2014.32.2.63 (2014).

16. Kim, T. H. et al. Role of adjuvant chemoradiotherapy for resected extrahepatic biliary tract cancer. Int J Radiat Oncol Biol Phys 81, e853-859, https://doi.org/10.1016/j.ijrobp.2010.12.019 (2011).

17. Nehls, O., Gregor, M. \& Klump, B. Serum and bile markers for cholangiocarcinoma. Semin Liver Dis 24, 139-154, https://doi. org/10.1055/s-2004-828891 (2004).

\section{Acknowledgements}

This work was supported by the National Research Foundation of Korea Grant funded by the Korean Government (number NRF-2016R1D1A1B03930229).

\section{Author Contributions}

J.H.I. and I.J.L. conceived this study. J.H.I. collected and organized the data. J.H.I., J.S.P. and I.J.L. analyzed the data and wrote the paper. D.S.Y., D.K.L. and J.W.K. provided data interpretation and edited the article. All authors reviewed the manuscript.

\section{Additional Information}

Competing Interests: The authors declare no competing interests.

Publisher's note: Springer Nature remains neutral with regard to jurisdictional claims in published maps and institutional affiliations.

(c) (i) Open Access This article is licensed under a Creative Commons Attribution 4.0 International License, which permits use, sharing, adaptation, distribution and reproduction in any medium or format, as long as you give appropriate credit to the original author(s) and the source, provide a link to the Creative Commons license, and indicate if changes were made. The images or other third party material in this article are included in the article's Creative Commons license, unless indicated otherwise in a credit line to the material. If material is not included in the article's Creative Commons license and your intended use is not permitted by statutory regulation or exceeds the permitted use, you will need to obtain permission directly from the copyright holder. To view a copy of this license, visit http://creativecommons.org/licenses/by/4.0/.

(C) The Author(s) 2019 